Open Access

Original Article

\title{
Cardiac cycle time-corrected electromechanical activation time greater than $15 \%$ is an independent risk factor for major adverse cardiovascular events in chronic heart failure outpatients
}

\author{
Jing Zhang ${ }^{1}$, Wenxian Liu²
}

\begin{abstract}
Objectives: This study aimed to investigate the predictive value of cardiac cycle time-corrected electromechanical activation time (EMATC) for major adverse cardiovascular events (MACEs) in outpatients with chronic heart failure (CHF) in comparison with other clinic indexes.

Methods: This prospective observational study at Beijing Anzhen Hospital from January 01, 2015 to January 012018 enrolled $120 \mathrm{CHF}$ patients who were admitted for acute onset of CHF and followed up after discharge for 616.5 days (range: 202.75-875.25 days). Based on the different endpoints, cardiogenic death, all-cause death, and HF-related readmission, patients were assigned to the following groups: cardiogenic death and non-cardiogenic death groups, all-cause death and survival groups, and HF readmission and nonreadmission groups. EMATc and other clinic indexes were measured and compared between these groups. Cox regression analysis was used to identify independent risk factors for MACEs.

Results: The hazard ratio for EMATc $>15 \%$ for cardiogenic death was 3.493 ( $P=0.046)$, suggesting that an EMATC $>15 \%$ was an independent risk factor for cardiogenic death in CHF patients. The hazard ratios for $B$-type natriuretic peptide (BNP) $>400 \mathrm{ng} / \mathrm{L}$ for all-cause death and CHF readmission were $3.810(\mathrm{P}=0.008)$ and $2.764(P=0.031)$, respectively. Thus, $B N P>400 \mathrm{ng} / \mathrm{L}$ was an independent risk factor for all-cause death and readmission for $\mathrm{CHF}$. $\mathrm{EF}<40 \%$ was not found to be a significant risk factor for MACEs.

Conclusions: BNP level can predict the risk for poor prognosis in CHF patients. EMATc $>15 \%$ is an independent risk factor for cardiogenic death and should be considered as a supplement to serum BNP level and other clinical indexes for predicting cardiogenic death in CHF outpatients.
\end{abstract}

KEYWORDS: Cardiac cycle time-corrected electromechanical activation time (EMATC), chronic heart failure (CHF), major adverse cardiovascular events (MACEs).

doi: https://doi.org/10.12669/pjms.38.3.4500

How to cite this:

Zhang J, Liu W. Cardiac cycle time-corrected electromechanical activation time greater than $15 \%$ is an independent risk factor for major adverse cardiovascular events in chronic heart failure outpatients. Pak J Med Sci. 2022;38(3):456-461. doi: https://doi.org/10.12669/pjms.38.3.4500

This is an Open Access article distributed under the terms of the Creative Commons Attribution License (http://creativecommons.org/licenses/by/3.0), which permits unrestricted use, distribution, and reproduction in any medium, provided the original work is properly cited.

1. Jing Zhang, $M M$

2. Wenxian Liu, MM

1,2: Division of Cardiology, Coronary Care Unit, Beijing Anzhen Hospital, Capital Medical University, Beijing 100029, P.R. China.

Correspondence:

Wenxian Liu

Division of Cardiology, Coronary Care Unit, Beijing Anzhen Hospital, Capital Medical University, No. 2 Anzhen Road, Chaoyang District,

Beijing 100029, P.R. China.

E-mail: 18911662882@163.com

* Received for Publication:

* $1^{\text {st }}$ Revision Received:

* $2^{\text {nd }}$ Revision Received:

* Final Revision Accepted:
March 30, 2021

May 6, 2021

Ocotber 27, 2021

November 15, 2021

\section{INTRODUCTION}

Chronic heart failure (CHF) is the end stage of a variety of cardiovascular diseases. The high CHF mortality is mainly attributed to complications of cardiovascular etiology. ${ }^{1}$ Despite medical advances, the all-cause mortality of CHF is $17 \%$, and the readmission rate is $44 \% .^{2}$ Searching for predictors of poor prognosis in CHF patients has been an active area of research. Natriuretic peptides such as B-type natriuretic peptide (BNP) have been shown to have diagnostic and predictive value for $\mathrm{CHF}^{3}$ and left ventricular 
ejection fraction (EF) is the most commonly used prognostic indicator for $\mathrm{CHF}^{4}$ However, for stable CHF patients, repeated monitoring of $\mathrm{BNP}$ and echocardiography require considerable medical resources and increases the burden on society and the patients' families. ${ }^{5}$

Acoustic cardiography is a rapid bedside test that can provide parameters reflecting left ventricular function. Electromechanical activation time (EMAT) is one of those parameters and refers to the time from the beginning of LV electrical activity (the beginning of QRS wave in electrocardiograph) to the beginning of the first heart sound (mitral valve closure). ${ }^{6}$ EMAT is divided by the cardiac cycle (RR interval) and can be compared for different heart rates (corrected EMAT ratio, EMATc). Elevated EMATc is considered as an indicator of decompensated HF. ${ }^{7}$ However, whether EMATc can be predictive for major adverse cardiovascular events (MACEs) in CHF outpatient remains to be examined. In the present study, we selected patients with CHF and investigated whether the EMATc can reliably be used to predict MACEs in patients with stable CHF in comparison to the traditional clinical indexes such as BNP and EF.

\section{METHODS}

Patient selection: Patients admitted to Beijing Anzhen Hospital from January 01, 2015 to January 01, 2018 for acute onset of CHF were enrolled in our study. They were admitted for worsening symptoms, which were not relieved by oral treatment.

The inclusion criteria included: $\mathrm{EF}<50 \%$, heart function classification of III-IV (New York Heart Association [NYHA]), and willingness to complete follow-up.

The exclusion criteria were: age $<18$ years, acute myocarditis, acute myocardial infarction, plans for coronary intervention or cardiac surgery, and structural heart disease.

After application of the inclusion and exclusion criteria, these patients received standard treatment. The study protocol used in this study was approved by the Ethics Committee of Beijing Anzhen Hospital, Capital Medical University (Approval No. 2019042X). All procedures performed in this study involving human participants met the ethical standards of the institutional and national research committee and the 1964 Declaration of Helsinki and its later amendments or comparable ethical standards. Written informed consent was obtained from each study participant.
Data Collection: All participants' baseline data, including age, gender, medications, medical history, and MACEs that occurred during the follow-up period, were collected. The clinical data of all participants included blood pressure, resting heart rate (HR), and the levels of troponin-I (TnI), BNP and creatinine. BNP, TnI and creatinine were collected 24-48 hours before discharge. HR was measured after rest with patients in a supine position for 5-10 minutes or longer at 24 hours before discharge.

Acoustic cardiography: EMATc was measured 24 hours before discharge. After rest in a supine position for 5-10 minutes, patients underwent acoustic cardiography (AUDICOR, Inovise Medical, Inc., Portland, OR, USA). EMATc was calculated by a computerized algorithm using simultaneous ECG and heart sound data obtained from the V3/V4 standard precordial position. Three independent readings were obtained for each patient, and average values were used in this study.

Echocardiography: EF and LV end-diastolic diameter (LVEDD) were collected 24 hours before discharge and measured with the modified biplane Simpson's rule (PHILIPS Affiniti 50). ${ }^{8}$ The researcher who interpreted the echocardiographic observations was blinded to all acoustic cardiographic observations and clinical data.

Follow-up and endpoints: Of the 145 enrolled patients, 23 had cardiac death in hospital, one refused follow up, and one was lost to followup. All 120 included patients were followed up periodically by telephone call or out-patient visit every 2-3 months after discharge, and clinic visits for new symptoms were documented. Overall, the patients were followed up for 616.5 days (range: 202.75-875.25 days). The endpoints included cardiogenic death, all-cause death, and HF readmission. The definition of cardiogenic death included mortality caused by acute myocardial infarction or acute decompensated CHF that developed into cardiogenic shock or ventricular tachycardia/fibrillation. Sudden death occurring out of hospital was also included in cardiogenic death. Mortality for any reason was included in all-cause death. The definition of HF readmission included symptoms such as dyspnea, shortness of breath, ascites and chest pain that aggravated even after administration of oral diuretics, and acute coronary syndrome needing hospitalization. Patients were assigned to different groups based on the different endpoints. 
Statistical analysis: All statistical analyses were performed using SPSS, version 22.0 (SPSS, Inc., Chicago, IL, USA). Normally distributed continuous data are presented as mean \pm standard deviation (SD). Data with a non-normal distribution are presented as median with interquartile range [M (Q1, Q3)]. Continuous variables were compared with Mann-Whitney $U$ test or two-sample Student's t-test. Categorical variables are presented as proportion (frequency) and were compared with the Chi-square test. Independent risk factors for MACEs were identified by Cox regression analysis. EF and BNP were dichotomized with the upper normal limit, and Kaplan-Meier method was used to draw survival curves. P-values $<0.05$ were regarded as statistically significant.

Sample size calculation: according to methods reported in the literature, ${ }^{7,9}$ the EMATc of decompensated CHF is $15 \%$. The all-cause death and admission rates of patients with stable CHF in 12 months were $17 \%$ and $44 \%$, respectively. According to MERIT HF (the Metoprolol CR/XL Randomized Intervention Trial in congestive heart failure) post hoc analysis, the cardiac death rate with CHF NYHA II was $64 \%$. We used a significance level a of 0.05 , power (1- $\beta$ ) of 0.8 , and two-sided test for power calculation. We planned to enroll 106 outpatients in the final study. Considering the possibility that $10 \%$ patients would drop out, we finally enrolled 120 patients.

\section{RESULTS}

A total of 120 patients with an average age of 58.47 \pm 15.80 years were enrolled in this study. Among these patients, 14 patients had cardiogenic death, and 11 died from a non-cardiac cause. Hence, the total number of all-cause deaths was 25 . Twentythree patients were readmitted for acute onset of HF. Comparison of demographic data between different groups according to the endpoint is shown in Table-I.

No significant differences in demographic and clinical parameters were observed between the cardiogenic death and non-cardiogenic death groups. The all-cause death group had a significantly higher mean age and a higher proportion with a past revascularization history than the surviving group. The readmission group had a significantly higher average age (66.61 \pm 11.95 years vs. $53.58 \pm 15.49$ years, $\mathrm{P} \leq 0.001)$, proportion of patients with chronic renal failure, and proportion of patients treated with diuretics than the non-readmission group.

Clinical data between different groups according to the endpoint. Table-I

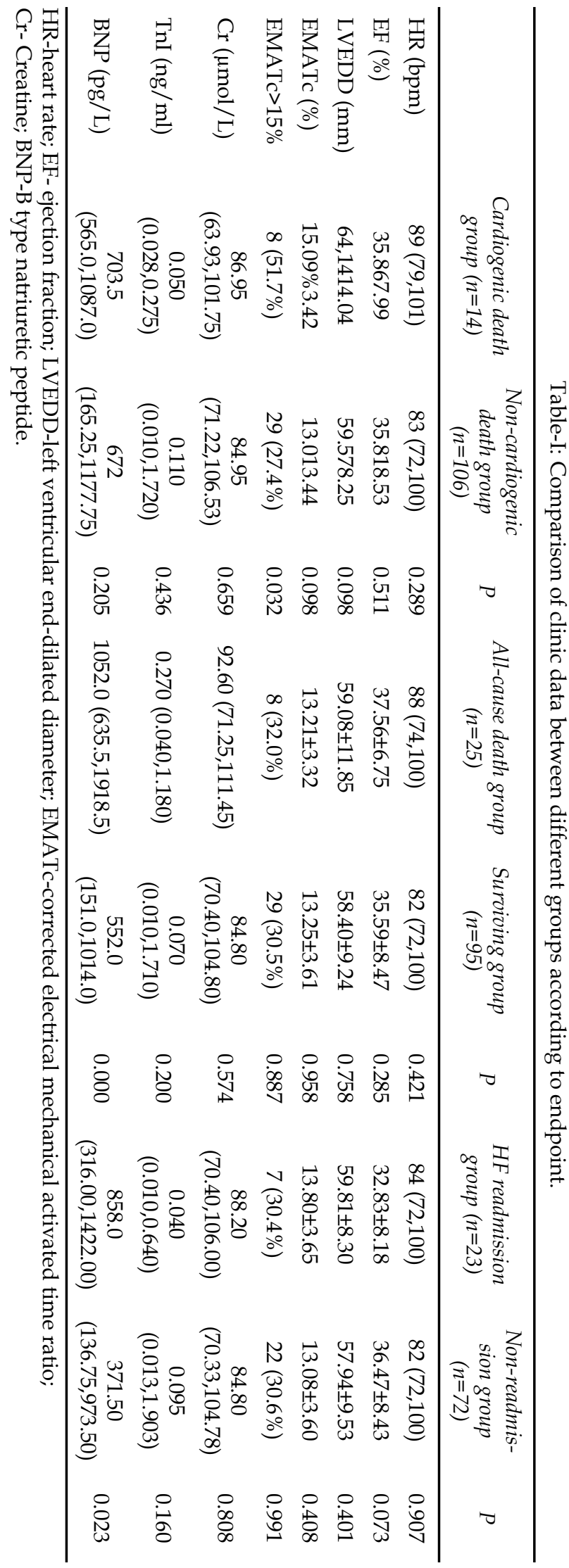

$\begin{array}{lllll}\text { Pak J Med Sci } & \text { March-April } 2022 & \text { Vol. } 38 & \text { No. } 3 & \text { www.pjms.org.pk } 458\end{array}$ 
Jing Zhang et al.

Table-II: Cox regression analysis of risk factors for MACEs.

\begin{tabular}{llccc}
\hline Endpoint & Factor & Hazard ratio & $95 \%$ CI & $P$ \\
\hline \multirow{3}{*}{ Cardogenic death } & EMATc $>15 \%$ & 3.493 & $1.021-11.947$ & 0.046 \\
& EF $<40 \%$ & 0.500 & $0.110-2.269$ & 0.369 \\
& BNP $>400 \mathrm{ng} / \mathrm{L}$ & 2.210 & $0.673-7.260$ & 0.191 \\
& EMATc $>15 \%$ & 1.021 & $0.414-2.515$ & 0.964 \\
All-cause death & EF $<40 \%$ & 0.739 & $0.273-2.001$ & 0.552 \\
& BNP $>400 \mathrm{ng} / \mathrm{L}$ & 3.810 & $1.409-10.300$ & 0.008 \\
& EMATc $>15 \%$ & 0.807 & $0.320-2.038$ & 0.650 \\
Readmission for $\mathrm{HF}$ & $\mathrm{EF}<40 \%$ & 1.158 & $0.367-3.654$ & 0.802 \\
& $\mathrm{BNP}>400 \mathrm{ng} / \mathrm{L}$ & 2.746 & $1.095-6.885$ & 0.031 \\
\hline
\end{tabular}

The cardiogenic death group had a lower proportion of patients with an EMATc $>15 \%$ than the non-cardiogenic death group $[8(51.7 \%)$ vs. $29(27.4 \%), P=0.032]$. The all-cause death group had a significantly higher BNP level [1052.0 $(635.5,1918.5)$ vs. $552.0(151.0,1014.0), \mathrm{P}<0.001]$. The HF readmission group had a higher serum BNP level [858.0 (316.00, 1422.00) vs. 371.50 (136.75, 973.50), $\mathrm{P}=0.023$ ] than the non-readmission group. Determination of risk factors for MACEs

We performed Cox regression analysis with $\mathrm{EF}<40 \%, \mathrm{BNP}>400 \mathrm{ng} / \mathrm{L}$ and EMATc $>15 \%$ as variables to analyze the risk factors for cardiac death, all-cause death, and readmission due to HF. For cardiogenic death, only EMATc $>15 \%$ was an

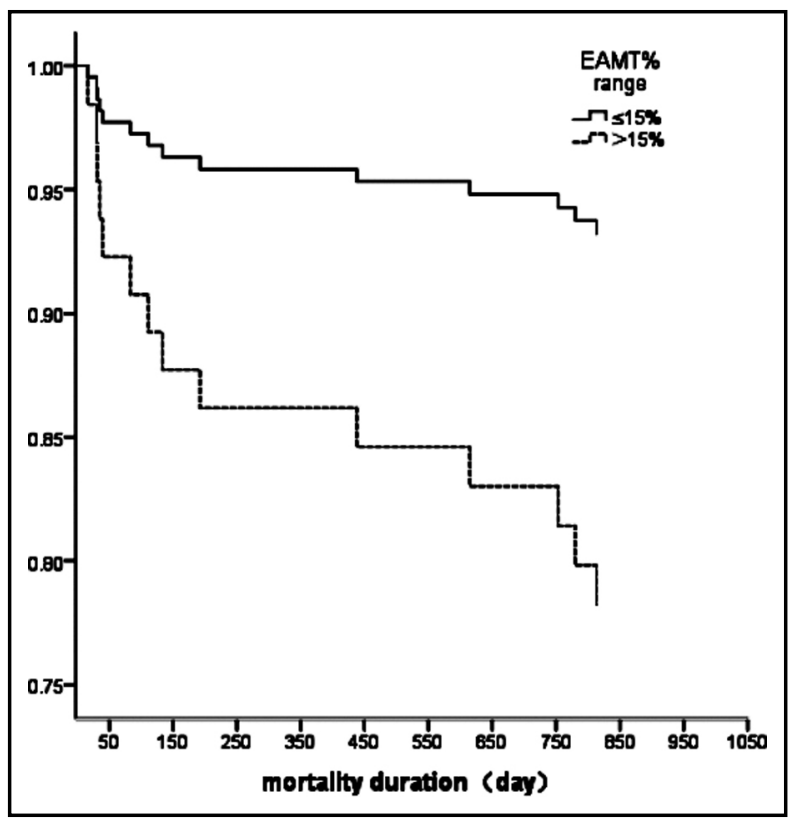

Fig.1: Survival curve for EMATc $>15 \%$ in relation to cardiogenic death in $\mathrm{CHF}$ patients. independent risk factor (Table-II). The survival curve for EMATC $>15 \%$ for cardiac death showed that the Log Rank $\chi^{2}$ value was $5.450(\mathrm{P}=0.02$; Fig.1). For all-cause death, only $\mathrm{BNP}>400 \mathrm{ng} / \mathrm{L}$ was an independent risk factor for all-cause death in CHF patients (Table-II). For HF readmission, only $\mathrm{BNP}>400 \mathrm{ng} / \mathrm{L}$ was an independent risk factor for readmission due to HF (Table-II).

\section{DISCUSSION}

In this study, we found no significant difference in the mean EMATc between the cardiogenic death and non-cardiogenic death groups; however, an EMATc $>15 \%$ was an independent risk factor for cardiac death in CHF patients, as revealed by Cox regression analysis and survival analysis.

EMAT is part of the pre-systolic phase and represents the time period needed for LV contraction to generate enough pressure to close the mitral valve. ${ }^{10}$ The EMATc is significantly prolonged in patients with decreased EF. Also, an EMATc $>15 \%$ has a specificity of $94 \%$ and a sensitivity of $44 \%$ for the diagnosis of $\mathrm{LV}$ dysfunction, and was used as a criterion for left ventricular dysfunction. ${ }^{6,11}$ Our former study showed that elevated EMATc is an independent risk factor for in-hospital cardiogenic death, cardiogenic shock, and HF progression. ${ }^{12}$

It is well established that if the level of natriuretic peptide increases by $25 \%$, the risk of MACEs and cardiac death increases substantially. ${ }^{13-15}$ Consistent with the above observations, we found that the BNP level was significantly higher in the all-cause death group and the HF readmission group than in the respective control groups. There was no significant difference in the BNP level between the cardiogenic death and non-cardiogenic death 


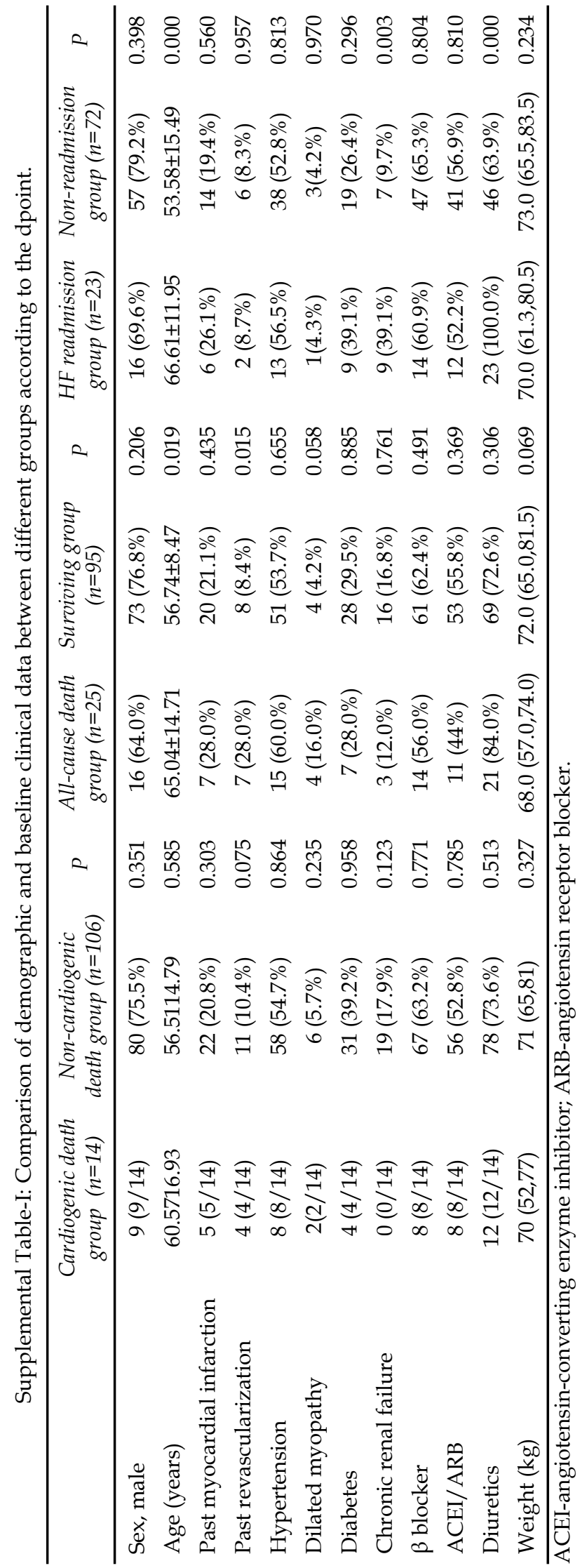

groups. Thus, it is highly recommended that EMATC $>15 \%$ be used as a supplementary tool to evaluate the risk of cardiogenic death in $\mathrm{CHF}$ patients.

BNP levels are affected by many factors including old age and renal insufficiency. ${ }^{16-18}$ The ages of patients in the HF readmission group and all-cause death group were higher. CHF patients younger than 50 years have a $<1 \%$ mortality rate, while those older than 80 years have an approximately 30\% mortality rate. ${ }^{19}$ Consistent with the above findings, in the present study, the patients in the all-cause death group were older than those in the survival group, and the patients with HF readmission were older than the patients without readmission.

Resting echocardiography is still the most commonly used cardiac imaging method for CHF patients. ${ }^{4}$ Elevation of the EF is the most important imaging parameter that predicts better outcomes in CHF patients. ${ }^{20}$ In the present study, there was no significant difference in EF between the cardiogenic death and non-cardiogenic death groups. This discrepancy was likely due to a difference in patient selection. It is possible that there was bias related to the population selection. The average EF of our patients was already lower than that of patients in a previous study. ${ }^{7}$

Limitations of this study: First, this study had a limited sample size, and thus, further studies are needed to corroborate our findings. Second, the follow-up duration was short. Third, some followups were made by phone, making it difficult to monitor EMATc, BNP or EF for further comparison. Fourth, the enrolled patients included only those with an $\mathrm{EF}<50 \%$; this study did not include patients with HF preserved ejection fraction (HFpEF). Fifth, the study was conducted between January 01, 2015 and January 01, 2018, during which time the angiotensin receptor-neprilysin inhibitor (ARNI) was not widely used in China. Therefore, we did not analyze the influence of the ARNI on the outcomes of CHF patients in this study.

\section{CONCLUSION}

In conclusion, an EMATc $>15 \%$ is an independent risk factor for cardiac death in stable CHF patients, can be used to predict the prognosis of stable $\mathrm{CHF}$ patients.

Clinical Trial Registration: http://www.chictr. org.cn/edit.aspx?pid=36214\&htm $=4$

Unique identifier: ChiCTR1900021470. 
Conflicts of Interest: None.

\section{Source of Funding: None.}

\section{REFERENCES}

1. Pocock SJ, Ariti CA, McMurray JJ, Maggioni A, Kober L, Squire IB, et al. Predicting survival in heart failure: a risk score based on 39372 patients from 30 studies. Eur Heart J. 2013;34(19):1404-1413. doi: 10.1093/eurheartj/ehs337

2. Lund LH, Benson L, Dahlstrom U, Edner M, Friberg L. Association between use of beta-blockers and outcomes in patients with heart failure and preserved ejection fraction. JAMA. 2014;312(19):2008-2018. doi: 10.1001/ jama.2014.15241

3. Chow SL, Maisel AS, Anand I, Bozkurt B, de Boer RA, Felker GM, et al. Role of Biomarkers for the Prevention, Assessment, and Management of Heart Failure: A Scientific Statement From the American Heart Association. Circulation. 2017;135(22):e1054-e1091. doi: 10.1161/ cir.0000000000000490

4. Wagholikar KB, Fischer CM, Goodson A, Herrick CD, Rees M, Toscano E, et al. Extraction of Ejection Fraction from Echocardiography Notes for Constructing a Cohort of Patients having Heart Failure with reduced Ejection Fraction (HFrEF). J Med Syst. 2018;42(11):209. doi: 10.1007/ s10916-018-1066-7

5. Braga JR, Leong-Poi H, Rac VE, Austin PC, Ross HJ, Lee DS. Trends in the Use of Cardiac Imaging for Patients With Heart Failure in Canada. JAMA Netw Open. 2019;2(8):e198766. doi: 10.1001/jamanetworkopen.2019.8766

6. Erne P, Resink TJ, Mueller A, Coslovsky M, Kobza R, Conen $\mathrm{D}$, et al. Use of acoustic cardiography immediately following electrical cardioversion to predict relapse of atrial fibrillation. J Atr Fibrillation. 2017;10(1):1527. doi: 10.4022/jafib.1527

7. Chao TF, Sung SH, Cheng HM, Yu WC, Wang KL, Huang $\mathrm{CM}$, etal. Electromechanical activation timein the prediction of discharge outcomes in patients hospitalized with acute heart failure syndrome. Intern Med. 2010;49(19):2031-2037. doi: 10.2169/internalmedicine.49.3944

8. Younis A, Mulla W, Goldkorn R, Klempfner R, Peled Y, Arad M, et al. Differences in Mortality of New-Onset (DeNovo) Acute Heart Failure Versus Acute Decompensated Chronic Heart Failure. Am J Cardiol. 2019;124(4):554-559. doi: 10.1016/j.amjcard.2019.05.031

9. Roos M, Toggweiler S, Zuber M, Jamshidi P, Erne P. Acoustic cardiographic parameters and their relationship to invasive hemodynamic measurements in patients with left ventricular systolic dysfunction. Congest Heart Fail. 2006;12(Suppl1):119-124. doi: 10.1111/j.15275299.2006.05769.x
10. Diamant B, Killip T. Indirect assessment of left ventricular performance in acute myocardial infarction. Circulation. 1970;42(4):579-592. doi: 10.1161/01.cir.42.4.579

11. Burkhoff D, Bailey G, Gimbel JR. Characterization of cardiac acoustic biomarkers in patients with heart failure. Ann Noninvasive Electrocardiol. 2020;25(2):e12717. doi: 10.1111/anec.12717

12. Zhang J, Liu WX, Lyu SZ. Predictive Value of Electromechanical Activation Time for In-Hospital Major Cardiac Adverse Events in Heart Failure Patients. Cardiovasc Ther. 2020;20204532596. doi: 10.1155/2020/4532596

13. Glick D, deFilippi CR, Christenson R, Gottdiener JS, Seliger SL. Long-term trajectory of two unique cardiac biomarkers and subsequent left ventricular structural pathology and risk of incident heart failure in community-dwelling older adults at low baseline risk. JACC Heart Fail. 2013;1(4):353360. doi: 10.1016/j.jchf.2013.04.007

14. McLellan J, Heneghan CJ, Perera R, Clements AM, Glasziou PP, Kearley KE, et al. B-type natriuretic peptideguided treatment for heart failure. Cochrane Database Syst Rev. 2016;12Cd008966. doi: 10.1002/14651858. CD008966.pub2

15. Richards AM, Troughton RW. Use of natriuretic peptides to guide and monitor heart failure therapy. Clin Chem. 2012;58(1):62-71. doi: 10.1373/clinchem.2011.165704

16. Carubelli V, Cotter G, Davison B, Gishe J, Senger S, Bonadei $\mathrm{I}$, et al. In-hospital worsening heart failure in patients admitted for acute heart failure. Int J Cardiol. 2016;225:353361. doi: 10.1016/j.ijcard.2016.10.002

17. Gupta DK, Wang TJ. Natriuretic Peptides and Cardiometabolic Health. Circ J. 2015;79(8):1647-1655. doi: 10.1253/circj.CJ-15-0589

18. Zois NE, Bartels ED, Hunter I, Kousholt BS, Olsen LH, Goetze JP. Natriuretic peptides in cardiometabolic regulation and disease. Nat Rev Cardiol. 2014;11(7):403412. doi: $10.1038 /$ nrcardio.2014.64

19. Yancy CW, Jessup M, Bozkurt B, Butler J, Casey DE, Jr., Drazner $\mathrm{MH}$, et al. ACCF/AHA guideline for the management of heart failure: a report of the American College of Cardiology Foundation/American Heart Association Task Force on Practice Guidelines. J Am Coll Cardiol. 2013;62(16):e147-e239. doi: 10.1016/j. jacc.2013.05.019

20. Kramer DG, Trikalinos TA, Kent DM, Antonopoulos GV, Konstam MA, Udelson JE. Quantitative evaluation of drug or device effects on ventricular remodeling as predictors of therapeutic effects on mortality in patients with heart failure and reduced ejection fraction: A metaanalytic approach. J Am Coll Cardiol. 2010;56(5):392-406. doi: $10.1016 / j$ j.jacc.2010.05.011 